\title{
Bounce and cyclic cosmology in new gravitational scalar-tensor theories
}

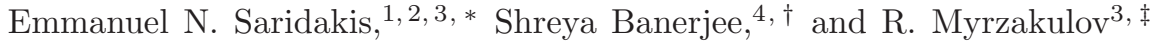 \\ ${ }^{1}$ Chongqing University of Posts 85 Telecommunications, Chongqing, 400065, China \\ ${ }^{2}$ Department of Physics, National Technical University of Athens, Zografou Campus GR 157 73, Athens, Greece \\ 3 Eurasian International Center for Theoretical Physics, \\ Eurasian National University, Astana 010008, Kazakhstan \\ ${ }^{4}$ Tata Institute of Fundamental Research, Homi Bhabha Road, Mumbai 400005, India
}

\begin{abstract}
We study the bounce and cyclicity realization in the framework of new gravitational scalartensor theories. In these theories the Lagrangian contains the Ricci scalar and its first and second derivatives, in a specific combination that makes them free of ghosts, and transformed into the Einstein frame they are proved to be a subclass of bi-scalar extensions of general relativity. We present analytical expressions for the bounce requirements, and we examine the necessary qualitative behavior of the involved functions that can give rise to a given scale factor. Having in mind these qualitative forms, we reverse the procedure and we construct suitable simple Lagrangian functions that can give rise to a bounce or cyclic scale factor.
\end{abstract}

PACS numbers: 98.80.-k, 95.36.+x, 04.50.Kd

\section{INTRODUCTION}

Although inflation is considered to be an important part of the universe history [1], the "problem of the initial singularity" is still present in the standard model of the universe. In particular, since such a singularity is unavoidable if inflation is driven by a scalar field in the framework of general relativity [2], a lot of effort has been devoted in resolving it through quantum gravity considerations or effective field theory applications.

Non-singular bouncing cosmologies may offer a potential solution to the cosmological singularity problem [3]. Modified gravities are an ideal framework for their realization, since they allow for the necessary violation of the null energy condition [4, 5]. In particular, one can obtain bouncing solutions in Pre-Big-Bang [6] and Ekpyrotic [7, 8] models, $f(R)$ gravity [9, 10], $f(T)$ gravity 11, gravity actions with higher order corrections 12, 13], braneworld scenarios 14, 15], non-relativistic gravity 16, 17], Lagrange modified gravity [18], massive gravity [19], loop quantum cosmology [20 22] etc. Alternatively, bouncing cosmology can be realized introducing matter fields that violate the null energy condition 23 25], or constructing non-conventional mixing terms [26, 27]. Furthermore, one may extend bouncing cosmology to the paradigm of cyclic cosmology [28], in which the universe experiences a sequence of expansions and contractions [29, 30] (see [34] for a review). This offers alternative insights for the origin of the observable universe 31 33], and can explain the scale invariant power spectrum [34, 35] and possible non-Gaussianities [36].

One recently constructed class of modified gravity is the so-called "new gravitational scalar-tensor theories"

\footnotetext{
*Electronic address: Emmanuel_Saridakis@baylor.edu

${ }^{\dagger}$ Electronic address: shreya.banerjee@tifr.res.in

‡Electronic address: rmyrzakulov@gmail.com
}

37, 38]. In these theories one uses a Lagrangian with the Ricci scalar and its first and second derivatives, however in a specific combination that makes the theory free of ghosts. Transforming into the Einstein frame, one can show that these constructions propagate $2+2$ degrees of freedom, and thus they fall outside Horndeski 39], Galileon [40, 41] and beyond Horndeski theories [42]. Nevertheless, although these theories can be seen as a subclass of bi-scalar extensions of general relativity, they can still be expressed in pure geometrical terms, and that is why the authors called them "new gravitational scalartensor theories". Due to the presence of extra degrees of freedom, these theories can lead to very interesting cosmological behavior [38].

In the present work, we are interested in studying the realization of bounce and cyclicity in the framework of new gravitational scalar-tensor theories. The plan of the work is as follows: In Section $\amalg$ we review the new gravitational scalar-tensor theories, and we apply them in a cosmological framework. In Section III we construct specific subclasses of Lagrangian functions that give rise to bouncing and cyclic scale factors. Finally, we summarize our results in section IV

\section{NEW GRAVITATIONAL SCALAR-TENSOR THEORIES}

In this section we briefly review the gravitational theories that include higher derivative curvature terms, which were named new gravitational scalar-tensor theories in 37]. Transformed into the Einstein frame these theories present two extra scalar degrees of freedom comparing to general relativity, and thus they fall in the class of bi-scalar modifications.

The action of the new gravitational scalar-tensor theories is

$$
S=\int d^{4} \sqrt{-g} f\left(R,(\nabla R)^{2}, \square R\right)
$$


with $(\nabla R)^{2}=g^{\mu \nu} \nabla_{\mu} R \nabla_{\nu} R$, and where for simplicity, here and in the following, we set the Planck mass $M_{p l}$ to one. Despite the presence of higher derivatives, these actions are ghost free and can be transformed into bi-scalar theories in the Einstein frame through double Lagrange multipliers. Although one can consider models, namely $f$-forms, nonlinear in $\square R=\beta$, in the present work we focus on theories with 38

$$
f\left(R,(\nabla R)^{2}, \square R\right)=\mathcal{K}\left(\left(R,(\nabla R)^{2}\right)+\mathcal{G}\left(R,(\nabla R)^{2}\right) \square R .\right.
$$

In this case, action (11) can be transformed to

$$
\begin{aligned}
S=\int d^{4} x \sqrt{-\hat{g}} & {\left[\frac{1}{2} \hat{R}-\frac{1}{2} \hat{g}^{\mu \nu} \nabla_{\mu} \chi \nabla_{\nu} \chi\right.} \\
& -\frac{1}{\sqrt{6}} e^{-\sqrt{\frac{2}{3}} \chi} \hat{g}^{\mu \nu} \mathcal{G} \nabla_{\mu} \chi \nabla_{\nu} \phi+\frac{1}{4} e^{-2 \sqrt{\frac{2}{3}} \chi} \mathcal{K} \\
& \left.+\frac{1}{2} e^{-\sqrt{\frac{2}{3}} \chi} \mathcal{G} \hat{\square} \phi-\frac{1}{4} e^{-\sqrt{\frac{2}{3}} \chi} \phi\right]
\end{aligned}
$$

where

$$
\mathcal{K}=\mathcal{K}(\phi, B), \quad \mathcal{G}=\mathcal{G}(\phi, B),
$$

with

$$
B=2 e^{\sqrt{\frac{2}{3}} \chi} g^{\mu \nu} \nabla_{\mu} \phi \nabla_{\nu} \phi .
$$

In the above expressions we have introduced the $\chi$-field through the conformal transformation $g_{\mu \nu}=\frac{1}{2} e^{-\sqrt{\frac{2}{3}}} \hat{g}_{\mu \nu}$ (the hat denotes the conformally related frame), while the $\phi$-field enters through $\varphi \equiv f_{\beta}$. We mention here that the above action contains two scalar fields, i.e. $\chi$ and $\phi$, however in the specific combination that makes it equivalent to the original higher-derivative gravitational action. Thus, these theories consist pure gravitational formulations of standard multi-scalar-tensor theories.

We will work in the Einstein-frame version of the above theories, namely with action (3), and for simplicity we neglect the hats. Variation of (3) with respect to the metric leads to the field equations

$$
\begin{aligned}
\mathcal{E}_{\mu \nu}= & \frac{1}{2} G_{\mu \nu}+\frac{1}{4} g_{\mu \nu} g^{\alpha \beta} \nabla_{\alpha} \chi \nabla_{\beta} \chi-\frac{1}{2} \nabla_{\mu} \chi \nabla_{\nu} \chi \\
& +\frac{1}{4} g_{\mu \nu} \sqrt{\frac{2}{3}} e^{-\sqrt{\frac{2}{3}} \chi} g^{\alpha \beta} \mathcal{G} \nabla_{\alpha} \chi \nabla_{\beta} \phi \\
& -\frac{1}{2} \sqrt{\frac{2}{3}} e^{-\sqrt{\frac{2}{3}} \chi} \mathcal{G} \nabla_{(\mu} \chi \nabla_{\nu)} \phi \\
& -\sqrt{\frac{2}{3}} g^{\alpha \beta} \nabla_{\alpha} \chi \nabla_{\beta} \phi \mathcal{G}_{B} \nabla_{\mu} \phi \nabla_{\nu} \phi \\
& -\frac{1}{4} g_{\mu \nu} e^{-\sqrt{\frac{2}{3}}} \chi \mathcal{G} \square \phi+\mathcal{G}_{B}(\square \phi) \nabla_{\mu} \phi \nabla_{\nu} \phi \\
& +\frac{1}{2} e^{-\sqrt{\frac{2}{3}} \chi} \mathcal{G}_{\mu} \nabla_{\nu} \phi-\frac{1}{2} \nabla_{\kappa}\left(e^{-\sqrt{\frac{2}{3}} \chi} \mathcal{G}_{(\mu}^{\lambda} \delta_{\nu)}^{\kappa} \nabla_{\lambda} \phi\right) \\
& +\frac{1}{4} \nabla_{\kappa}\left(e^{-\sqrt{\frac{2}{3}} \chi} \mathcal{G}_{\mu \nu} \nabla^{\kappa} \phi\right)-\frac{1}{8} g_{\mu \nu} e^{-2 \sqrt{\frac{2}{3}} \chi} \mathcal{K} \\
& +\frac{1}{2} e^{-\sqrt{\frac{2}{3}} \chi} \mathcal{K}_{B} \nabla_{\mu} \phi \nabla_{\nu} \phi+\frac{1}{8} g_{\mu \nu} e^{-\sqrt{\frac{2}{3}} \chi} \phi=0,
\end{aligned}
$$

where the parentheses in spacetime indices denote symmetrization, and the subscripts in $\mathcal{G}$ and $\mathcal{K}$ mark partial derivatives (e.g. $\mathcal{G}_{B}=\frac{\partial \mathcal{G}(\phi, B)}{\partial B}$ etc). Furthermore, variation of (3) with respect to $\chi$ and $\phi$ gives rise to their equations of motion, namely

$$
\begin{aligned}
\mathcal{E}_{\chi}= & \square \chi+\frac{1}{3} e^{-\sqrt{\frac{2}{3}} \chi} g^{\mu \nu} \mathcal{G} \nabla_{\mu} \chi \nabla_{\nu} \phi \\
& -\frac{2}{3} g^{\mu \nu} \nabla_{\mu} \chi \nabla_{\nu} \phi \mathcal{G}_{B} g^{\alpha \beta} \nabla_{\alpha} \phi \nabla_{\beta} \phi \\
& +\frac{1}{2} \sqrt{\frac{2}{3}} \nabla_{\mu}\left(e^{-\sqrt{\frac{2}{3}} \chi} g^{\mu \nu} \mathcal{G} \nabla_{\nu} \phi\right) \\
& -\frac{1}{2} \sqrt{\frac{2}{3}} e^{-\sqrt{\frac{2}{3}} \chi} \mathcal{G} \square \phi+\sqrt{\frac{2}{3}} \mathcal{G}_{B} \nabla_{\mu} \phi \nabla_{\nu} \phi g^{\mu \nu} \square \phi \\
& -\frac{1}{2} \sqrt{\frac{2}{3}} e^{-2 \sqrt{\frac{2}{3}} \chi} \mathcal{K}+\frac{1}{2} e^{-\sqrt{\frac{2}{3}} \chi} \mathcal{K}_{B} \sqrt{\frac{2}{3}} g^{\mu \nu} \nabla_{\mu} \phi \nabla_{\nu} \phi \\
& +\frac{1}{4} \sqrt{\frac{2}{3}} e^{-\sqrt{\frac{2}{3}} \chi} \phi=0,
\end{aligned}
$$

and

$$
\begin{aligned}
& \mathcal{E}_{\phi}=-\frac{1}{2} \sqrt{\frac{2}{3}} e^{-\sqrt{\frac{2}{3}} \chi} g^{\mu \nu} \mathcal{G}_{\phi} \nabla_{\mu} \chi \nabla_{\nu} \phi \\
& +2 \sqrt{\frac{2}{3}} \nabla_{\beta}\left(g^{\mu \nu} \mathcal{G}_{B} g^{\alpha \beta} \nabla_{\alpha} \phi \nabla_{\mu} \chi \nabla_{\nu} \phi\right) \\
& +\frac{1}{2} \sqrt{\frac{2}{3}} \nabla_{\nu}\left(e^{-\sqrt{\frac{2}{3}} \chi} g^{\mu \nu} \mathcal{G} \nabla_{\mu} \chi\right) \\
& +\frac{1}{2} e^{-\sqrt{\frac{2}{3}} x} \mathcal{G}_{\phi} \square \phi-2 \mathcal{G}_{B}(\square \phi)^{2}-2 \nabla_{\nu} \mathcal{G}_{B} \square \phi \nabla^{\nu} \phi \\
& -\frac{1}{2} \sqrt{\frac{2}{3}} \nabla^{\mu}\left(e^{-\sqrt{\frac{2}{3}} \chi} \nabla_{\mu} \chi \mathcal{G}\right)+\frac{1}{2} \nabla^{\mu}\left(e^{-\sqrt{\frac{2}{3}} \chi} \mathcal{G}_{\phi} \nabla_{\mu} \phi\right) \\
& -\frac{1}{2} \sqrt{\frac{2}{3}} e^{-\sqrt{\frac{2}{3}} \chi} \nabla^{\mu} \chi \mathcal{G}_{B} \nabla_{\mu} B+\frac{1}{2} e^{-\sqrt{\frac{2}{3}} \chi} \nabla^{\mu} \mathcal{G}_{B} \nabla_{\mu} B \\
& +\sqrt{\frac{2}{3}} e^{-\sqrt{\frac{2}{3}} \chi} \mathcal{G}_{B} \nabla^{\mu}\left(e^{\sqrt{\frac{2}{3}} \chi} \nabla_{\mu} \chi \nabla^{\nu} \phi \nabla_{\nu} \phi\right) \\
& +2 e^{-\sqrt{\frac{2}{3}} \chi} \mathcal{G}_{B} \nabla^{\mu}\left(e^{\sqrt{\frac{2}{3}} \chi} \nabla^{\nu} \phi\right) \nabla_{\mu} \nabla_{\nu} \phi \\
& +2 \mathcal{G}_{B} R^{\mu \nu} \nabla_{\mu} \phi \nabla_{\nu} \phi+\frac{1}{4} e^{-2 \sqrt{\frac{2}{3}} \chi} \mathcal{K}_{\phi} \\
& -\nabla_{\nu}\left(e^{-\sqrt{\frac{2}{3}} \chi} \mathcal{K}_{B} g^{\mu \nu} \nabla_{\mu} \phi\right)-\frac{1}{4} e^{-\sqrt{\frac{2}{3}} \chi}=0 .
\end{aligned}
$$

As mentioned above, we do verify that all field equations do not contain problematic higher-derivative terms, and thus theory (11) is indeed healthy as it is constructed to be. Finally, note that in the scenario at hand general relativity is reproduced when $\mathcal{K}=\phi / 2$ and $\mathcal{G}=0$, and in this case the triviality of the conformal transformation gives $\chi=-\sqrt{\frac{3}{2}} \ln 2$.

We proceed by applying the above theories into a cosmological framework. We add the matter sector straightaway in the Einstein frame and we consider the total action $S_{t o t}=S+S_{m}$ [38]. Therefore, the metric field 
equations (6) become

$$
\mathcal{E}_{\mu \nu}=\frac{1}{2} T_{\mu \nu},
$$

with $T_{\mu \nu}=\frac{-2}{\sqrt{-g}} \frac{\delta S_{m}}{\delta g^{\mu \nu}}$ the energy-momentum tensor of the matter sector considered to correspond to a perfect fluid. Moreover, we consider a flat Friedmann-RobertsonWalker (FRW) geometry with metric

$$
d s^{2}=-d t^{2}+a(t)^{2} \delta_{i j} d x^{i} d x^{j},
$$

with $a(t)$ is the scale factor, and hence the two scalars are time-dependent only. With these considerations, equations (6) lead to the two Friedmann equations:

$$
\begin{aligned}
3 H^{2}- & \rho_{m}-\frac{1}{2} \dot{\chi}^{2}+\frac{1}{4} e^{-2 \sqrt{\frac{2}{3}} \chi} \mathcal{K} \\
+ & \frac{2}{3} \dot{\phi}^{2}[\dot{\phi}(\sqrt{6} \dot{\chi}-9 H)-3 \ddot{\phi}] \mathcal{G}_{B} \\
& -\frac{1}{2} e^{-\sqrt{\frac{2}{3}} \chi}\left[\dot{B} \dot{\phi} \mathcal{G}_{B}+\frac{\phi}{2}+\dot{\phi}^{2}\left(\mathcal{G}_{\phi}-2 \mathcal{K}_{B}\right)\right]=0, \\
3 H^{2} & +2 \dot{H}+p_{m}+\frac{1}{2} \dot{\chi}^{2}+\frac{1}{4} e^{-2 \sqrt{\frac{2}{3}} \chi} \mathcal{K} \\
& +\frac{1}{2} e^{-\sqrt{\frac{2}{3}} \chi}\left(-\frac{\phi}{2}+\dot{B} \dot{\phi} \mathcal{G}_{B}+\dot{\phi}^{2} \mathcal{G}_{\phi}\right)=0,
\end{aligned}
$$

with $B(t)=2 e^{\sqrt{\frac{2}{3}}} g^{\mu \nu} \nabla_{\mu} \phi \nabla_{\nu} \phi=-2 e^{\sqrt{\frac{2}{3}}} \dot{\phi}^{2}, H=\dot{a} / a$ the Hubble parameter, and where dots denote differentiation with respect to $t$. Additionally, we have introduced the energy density $\rho_{m}$ and pressure $p_{m}$ of the matter fluid. Similarly, the two scalar field equations (7) and (8) lead to the scalar evolution equations:

$$
\begin{aligned}
\mathcal{E}_{\chi}= & \ddot{\chi}+3 H \dot{\chi}-\frac{1}{3} \dot{\phi}^{2}[\dot{\phi}(3 \sqrt{6} H-2 \dot{\chi})+\sqrt{6} \ddot{\phi}] \mathcal{G}_{B} \\
& +\frac{1}{2 \sqrt{6}} e^{-\sqrt{\frac{2}{3}} \chi}\left[2 \dot{B} \dot{\phi} \mathcal{G}_{B}-\phi+2 \dot{\phi}^{2}\left(\mathcal{K}_{B}+\mathcal{G}_{\phi}\right)\right] \\
& +\frac{1}{\sqrt{6}} e^{-2 \sqrt{\frac{2}{3}} \chi} \mathcal{K}=0,
\end{aligned}
$$

and

$$
\begin{aligned}
\mathcal{E}_{\phi}= & \frac{1}{3} e^{-\sqrt{\frac{2}{3}} \chi}[\dot{\phi}(-9 H+\sqrt{6} \dot{\chi})-3 \ddot{\phi}] \mathcal{K}_{B} \\
+ & \frac{1}{6} \dot{B}\left\{3 e^{-\sqrt{\frac{2}{3}} \chi} \dot{B}+4 \dot{\phi}[\dot{\phi}(9 H-\sqrt{6} \dot{\chi})+3 \ddot{\phi}]\right\} \mathcal{G}_{B B} \\
+ & \frac{1}{3} e^{-\sqrt{\frac{2}{3}} \chi}[\dot{\phi}(9 H-\sqrt{6} \dot{\chi})+3 \ddot{\phi}] \mathcal{G}_{\phi} \\
+ & \left\{e^{-\sqrt{\frac{2}{3}} \chi} \dot{B} \dot{\phi}+\frac{2}{3} \dot{\phi}^{2}[\dot{\phi}(9 H-\sqrt{6} \dot{\chi})+3 \ddot{\phi}]\right\} \mathcal{G}_{B \phi} \\
- & e^{-\sqrt{\frac{2}{3}} \chi} \dot{\phi}^{2} \mathcal{K}_{B \phi}+\frac{1}{2} e^{-\sqrt{\frac{2}{3}} \chi} \dot{\phi}^{2} \mathcal{G}_{\phi \phi}-e^{-\sqrt{\frac{2}{3}} \chi} \dot{B} \dot{\phi} \mathcal{K}_{B B} \\
+ & {\left[\frac{4}{3} \dot{\phi}(9 H-2 \sqrt{6} \dot{\chi}) \ddot{\phi}-\frac{1}{\sqrt{6}} e^{-\sqrt{\frac{2}{3}} \chi} \dot{B} \dot{\chi}\right.} \\
& \left.+\dot{\phi}^{2}\left(18 H^{2}+6 \dot{H}-3 \sqrt{6} H \dot{\chi}-\frac{2}{3} \dot{\chi}^{2}-\sqrt{6} \ddot{\chi}\right)\right] \mathcal{G}_{B} \\
- & \frac{1}{4} e^{-2 \sqrt{\frac{2}{3}} \chi} \mathcal{K}_{\phi}+\frac{1}{4} e^{-\sqrt{\frac{2}{3}} \chi}=0,
\end{aligned}
$$

with $\mathcal{G}_{B \phi}=\mathcal{G}_{\phi B} \equiv \frac{\partial^{2} \mathcal{G}}{\partial B \partial \phi}$, etc. We mention that amongst the above four equations, namely (11),(12), (13), (14), only three are independent, due to the fact that the total action is diffeomorphism invariant, i.e. we have a conservation equation [38]:

$$
\nabla_{\mu} \mathcal{E}^{\mu \nu}+\frac{1}{2} \mathcal{E}_{\chi} \nabla^{\nu} \chi+\frac{1}{2} \mathcal{E}_{\phi} \nabla^{\nu} \phi=\frac{1}{2} \nabla_{\mu} T^{\mu \nu}=0 .
$$

Thus, the matter energy density and pressure satisfy the standard evolution equation

$$
\dot{\rho}_{m}+3 H\left(\rho_{m}+p_{m}\right)=0 .
$$

The investigation of the above cosmological scenario at late times was performed in [38], where it was indeed shown that one can obtain interesting phenomenology. In the present work we are interested in studying the earlytime phases, and in particular to examine whether the bounce realization is possible. This is performed in the following sections.

\section{BOUNCING AND CYCLIC SOLUTIONS}

In this section we proceed to the investigation of bounce and cyclicity realization in cosmology driven by new gravitational scalar-tensor theories. As it is known, in order to obtain a bounce we need a contracting phase $(H<0)$ succeeded by an expanding one $(H>0)$, and thus at the bounce point we have $H=0$ and $\dot{H}>0$. On the other hand, for the turnaround realization we need the succession of an expanding and a contracting phase, and thus at the turnaround point $H=0$ and $\dot{H}<0$. Although these conditions cannot be fulfilled in the framework of general relativity [13], observing the form of the two Friedmann equations (11), (12), as well as of the scalar-field equations (13), (14) we deduce that for suitable choices of the free functions $\mathcal{K}$ and $\mathcal{G}$ one can obtain the necessary violation of the null energy condition and thus satisfy the aforementioned bouncing and cyclic conditions.

\section{A. Reconstruction of a bounce}

Let us now proceed to the investigation of the bounce realization. Suppose that we impose a given form of a bouncing scale factor, in which case $H(t)$ and $\dot{H}(t)$ are also known. Substitution of this bouncing scale factor into the three independent equations (11), (13) and (14), and recalling that in FRW geometry $B(t)=$ $-2 e^{\sqrt{\frac{2}{3}} \chi(t)} \dot{\phi}(t)^{2}$, we obtain a system of three differential equations for the four functions $\phi(t)$ and $\chi(t)$ and for $\mathcal{K}$ and $\mathcal{G}$ (and their derivatives) considered as functions of $t$. Thus, we have the freedom to further consider the form of one of $\mathcal{K}$ and $\mathcal{G}$. In the following paragraphs we examine two such cases separately, having in mind that in order to be able to obtain a bounce we need to go beyond 
the simple $\mathcal{K}$ and $\mathcal{G}$ forms investigated in [38], which were adequate to describe the late-time universe.

\section{Model $I: \mathcal{K}=\phi / 2$ and $\mathcal{G}=\mathcal{G}(B)$}

Since general relativity is re-obtained for $\mathcal{K}=\phi / 2$ and $\mathcal{G}=0$, one class of viable models of new gravitational scalar-tensor theories is the one with

$$
\mathcal{K}=\frac{\phi}{2}
$$

and with

$$
\mathcal{G}=\mathcal{G}(B),
$$

i.e. $\mathcal{G}$ is independent of $\phi$. Concerning the explicit bouncing scale factor, without loss of generality we consider the matter bounce form [11]

$$
a(t)=a_{b}\left(1+q t^{2}\right)^{1 / 3},
$$

with $a_{b}$ denoting the value of scale factor at the bounce point $t=0$, and $q$ a positive parameter which determines how fast the bounce is realized. From this scale factor we immediately find

$$
\begin{aligned}
H(t) & =\frac{2 q t}{3\left(1+q t^{2}\right)} \\
\dot{H}(t) & =\frac{2 q}{3}\left[\frac{1-q t^{2}}{\left(1+q t^{2}\right)^{2}}\right] .
\end{aligned}
$$

Inserting these considerations into equations (11), (13) and (14), and replacing $\mathcal{G}_{B}(t)=\mathcal{G}^{\prime}(t) / B^{\prime}(t)$, and $\mathcal{G}_{B B}(t)=\left(\mathcal{G}^{\prime \prime}(t) B^{\prime}(t)-B^{\prime \prime}(t) \mathcal{G}^{\prime}\right) /\left(B^{\prime}(t)\right)^{3}$, we obtain a system of differential equations for $\phi(t), \chi(t)$ and $G(t)$. Unfortunately, this system cannot be solved analytically, however it can easily be elaborated numerically, leading to the extraction of the $\phi(t), \chi(t)$ and $G(t)$. Since $B(t)$ can then be found, we can acquire the function $\mathcal{G}(B)$ in a parametric form. Hence, it is this reconstructed $\mathcal{G}(B)$ that generates the input scale factor (19).

In Fig. 1 we present the $\mathcal{G}(B)$ that is reconstructed from the given bouncing scale-factor form (19), according to the above procedure, where we have neglected the matter sector in order to investigate the pure effect of the novel terms of the present theory. As we observe from the above procedure, and in particular from Fig. 1, in order to obtain a bouncing scale factor in the case where $\mathcal{K}=\phi / 2$, we need a $\mathcal{G}(B)$ function that resembles an exponential function of $B$.

The explicit example of the above reconstruction procedure offered us qualitative information for the $\mathcal{G}(B)$ form that leads to a bouncing scale factor. Thus, we can now reverse the reconstruction procedure and impose the form of $\mathcal{G}(B)$ a priori, and then extract the induced scale factor, which is the physical procedure. Having the qualitative requirements for $\mathcal{G}(B)$ in mind, we choose its form to be

$$
\mathcal{G}(B)=G_{0} e^{G_{1} B}
$$

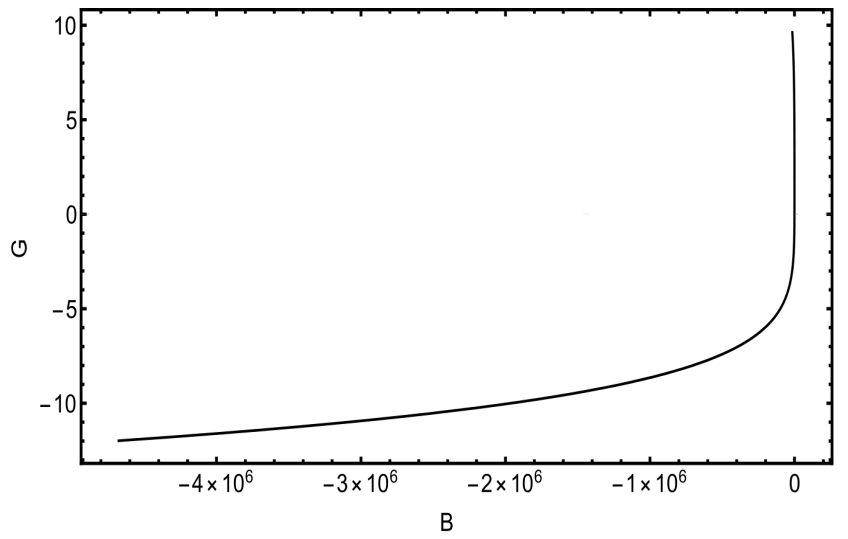

FIG. 1: The reconstructed $\mathcal{G}(B)$ that generates the bouncing scale factor 19), in the case where $\mathcal{K}=\phi / 2$. The bouncing parameters have been chosen as $a_{b}=0.2, q=10^{-5}$. All quantities are measured in $M_{p l}$ units.

where $G_{0}$ and $G_{1}$ are parameters. Substituting it into equations (11), (12) and (13), considering once again $\mathcal{K}=\phi / 2$, we acquire three second order differential equations for $a(t), \phi(t)$ and $\chi(t)$. Elaborating the system numerically we extract the scale factor, and we depict it in Fig. 2. Hence, we do verify that new gravitational

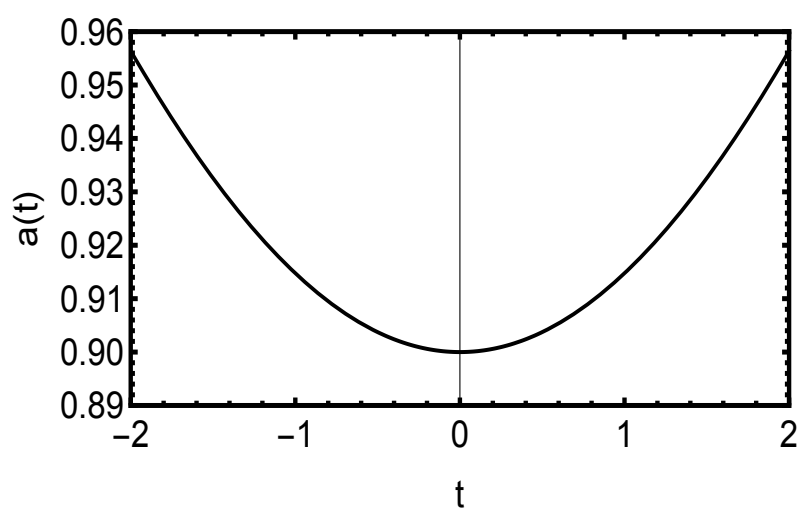

FIG. 2: The evolution of the scale factor a $(t)$ that is generated by the the exponential form $\mathcal{G}(B)=G_{0} e^{G_{1} B}$, in the case where $\mathcal{K}=\phi / 2$, with $G_{0}=-7$ and $G_{1}=0.0001$ in $M_{p l}$ units.

scalar-tensor theories with $\mathcal{K}=\phi / 2$ and $\mathcal{G}(B)=G_{0} e^{G_{1} B}$ lead to the realization of a cosmological bounce.

The above procedure can easily be repeated in the presence of the matter sector which gives rise to matter bounce. We find that the same forms of the functions $\mathcal{K}$ and $\mathcal{G}$ in the presence of pressureless matter, i.e. with $p_{m}=0$ and $\rho_{m} \propto a^{3}$, give rise to a matter bounce, namely to a scale-factor similar to that of Fig. 2. 
2. Model II: $\mathcal{K}=\phi / 2+f(B)$ and $\mathcal{G}=\xi B$

In this paragraph we investigate the bounce realization in a different subclass of new gravitational scalar-tensor theories. In particular, we choose

$$
\begin{aligned}
& \mathcal{K}=\frac{\phi}{2}+f(B) \\
& \mathcal{G}=\xi B,
\end{aligned}
$$

where $f(B)$ is an unknown function of $B$ and $\xi$ is a parameter. Similarly to the previous paragraph, firstly we consider the bounce scale factor (19), in order to numerically reconstruct $f(B)$ and acquire a qualitative picture of its form. Indeed, substituting these into equations (11), (13) and (14), and replacing $\mathcal{K}_{B}=f^{\prime}(t) / B^{\prime}(t)$ and $\mathcal{K}_{B B}(t)=\left(f^{\prime \prime}(t) B^{\prime}(t)-B^{\prime \prime}(t) f^{\prime}\right) /\left(B^{\prime}(t)\right)^{3}$, we obtain a system of differential equations for $\phi(t), \chi(t)$ and $f(t)$. Since this system cannot be solved analytically, we elaborate it numerically and we extract $\phi(t), \chi(t)$ and $f(t)$. Since $B(t)$ can then be found as $B(t)=-2 e^{\sqrt{\frac{2}{3}} \chi(t)} \dot{\phi}(t)^{2}$, we can finally acquire the function $f(B)$ in a parametric form. Hence, this reconstructed $f(B)$ generates the input scale factor (19).

In Fig. 3 we present the $f(B)$ that is reconstructed according to the above procedure, in the absence of matter sector. As we observe, in order to obtain a bouncing scale

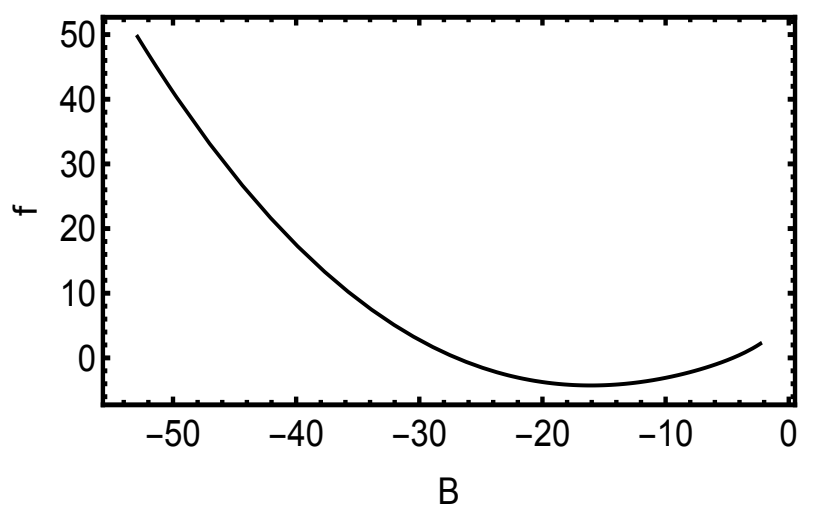

FIG. 3: The reconstructed $f(B)$ that generates the bouncing scale factor (19), in the case where $\mathcal{K}=\phi / 2+f(B)$ and $\mathcal{G}=$ $\xi B$. The bouncing parameters have been chosen as $a_{b}=0.2$, $q=10^{-5}$, while $\xi=0.1$. All quantities are measured in $M_{p l}$ units.

factor in the case where $\mathcal{K}=\phi / 2+f(B)$ and $\mathcal{G}=\xi B$, we need a $f(B)$ form that resembles a parabolic function of $B$.

Having in mind the qualitative information for the form of $f(B)$ obtained through the above reconstruction procedure, we can now reverse the procedure and impose the form of $f(B)$ a priori, and then extract the induced scale factor. We choose

$$
\mathcal{K}(\phi, B)=\phi / 2+\left(B-B_{0}\right)^{2},
$$

where $B_{0}$ is a constant. Substituting it into equations (11), (12) and (13), alongside with $\mathcal{G}=\xi B$, we result to three second order differential equations for $a(t), \phi(t)$ and $\chi(t)$. Elaborating the system numerically we extract the scale factor, which is presented in Fig. 4. Thus, we do verify that new gravitational scalar-tensor theories with $\mathcal{K}(\phi, B)=\phi / 2+\left(B-B_{0}\right)^{2}$ and $\mathcal{G}=\xi B$ lead to the realization of a cosmological bounce.

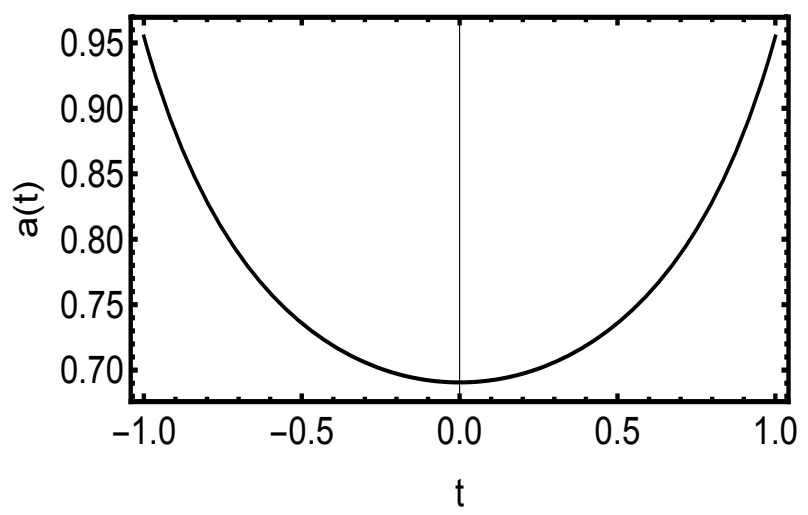

FIG. 4: The evolution of the scale factor a $(t)$ that is generated by $\mathcal{K}(\phi, B)=\phi / 2+\left(B-B_{0}\right)^{2}$ and $\mathcal{G}=\xi B$, with $B_{0}=25.5$ and $\xi=0.1$ in $M_{p l}$ units.

Finally, we mention that the above procedure can be repeated in the presence of the matter sector, and we find that the same forms of the functions $\mathcal{K}$ and $\mathcal{G}$ in the presence of dust matter give rise to a matter bounce.

\section{General conditions for a bounce}

We close this subsection by examining analytically the conditions for the bounce realization in the theories and models at hand, namely $H=0$ and $\dot{H}>0$ at the bounce point. In particular, the first Friedmann equation (11) provides the general equation satisfied by the Hubble function, namely

$$
H^{2}+b H+c=0
$$

where

$$
\begin{aligned}
b= & -2 \dot{\phi}^{3} \mathcal{G}_{B} \\
c= & -\frac{1}{6} \dot{\chi}^{2}+\frac{1}{12} e^{-2 \sqrt{\frac{2}{3}} \chi} \mathcal{K}+\frac{2}{9} \dot{\phi}^{2}(\dot{\phi} \sqrt{6} \dot{\chi}-3 \ddot{\phi}) \mathcal{G}_{B} \\
& -\frac{1}{6} e^{-\sqrt{\frac{2}{3}} \chi}\left[\dot{B} \dot{\phi} \mathcal{G}_{B}+\frac{\phi}{2}+\dot{\phi}^{2}\left(\mathcal{G}_{\phi}-2 \mathcal{K}_{B}\right)\right] .
\end{aligned}
$$

The general solution of the above quadratic equation is

$$
H=\frac{-b \pm \sqrt{b^{2}-12 c}}{6} .
$$

Hence, in order for the first bounce condition, namely $H_{b}=0$ (the subscript "b" denotes the value at the bounce point), to be satisfied, we deduce that at the bounce point we need $c=0$. In the case of Model I 
above, i.e. for $\mathcal{K}=\phi / 2$ and $\mathcal{G}=\mathcal{G}(B)$, this condition becomes

$$
\begin{aligned}
-3 \dot{\chi}^{2} & +\frac{3 \phi}{4} e^{-2 \sqrt{\frac{2}{3}} \chi}+4 \dot{\phi}^{2}(\dot{\phi} \sqrt{6} \dot{\chi} \\
& -3 \ddot{\phi}) \mathcal{G}_{B}-3 e^{-\sqrt{\frac{2}{3}} \chi}\left(\dot{B} \dot{\phi} \mathcal{G}_{B}+\frac{\phi}{2}\right)=0
\end{aligned}
$$

at the bounce point, while for Model II above, i.e. for $\mathcal{K}=\phi / 2+f(B)$ and $\mathcal{G}=\xi B$, this condition becomes

$$
\begin{aligned}
&-6 \dot{\chi}^{2}+3 e^{-2 \sqrt{\frac{2}{3}} \chi}\left[\frac{\phi}{2}+f(B)\right]+8 \dot{\phi}^{2}(\dot{\phi} \sqrt{6} \dot{\chi} \\
&-3 \ddot{\phi}) \xi-6 e^{-\sqrt{\frac{2}{3}} \chi}\left(\dot{B} \dot{\phi} \xi+\frac{\phi}{2}-2 \dot{\phi}^{2} f_{B}\right)=0
\end{aligned}
$$

at the bounce point. The above conditions simplify further once we consider the forms of the functions $\mathcal{G}(B)$ and $f(B)$ obtained above.

Concerning the second bounce condition, namely $\dot{H}>$ 0 at the bounce point, from Eq. (12) we acquire

$$
2 \dot{\chi}^{2}+e^{-2 \sqrt{\frac{2}{3}} \chi} \mathcal{K}+2 e^{-\sqrt{\frac{2}{3}} \chi}\left(\dot{B} \dot{\phi} \mathcal{G}_{B}-\frac{\phi}{2}+\dot{\phi}^{2} \mathcal{G}_{\phi}\right)>0 .
$$

Thus, in the cases of Model I and Model II respectively we obtain

$$
4 \dot{\chi}^{2}+e^{-2 \sqrt{\frac{2}{3}} \chi} \phi+4 e^{-\sqrt{\frac{2}{3}} \chi}\left(\dot{B} \dot{\phi} \mathcal{G}_{B}-\frac{\phi}{2}\right)>0
$$

and

$$
2 \dot{\chi}^{2}+e^{-2 \sqrt{\frac{2}{3}} \chi}\left[\frac{\phi}{2}+f(B)\right]+2 e^{-\sqrt{\frac{2}{3}} \chi}\left(\dot{B} \dot{\phi} \xi-\frac{\phi}{2}\right)>0 .
$$

We mention here that in the analysis of the previous paragraphs we took the above general requirements into account in order to determine the initial conditions for the differential equations at the bounce point. Indeed, as we mentioned above, in order to be able to satisfy the above requirements and obtain a bounce, one must go beyond the simple $\mathcal{K}$ and $\mathcal{G}$ forms of [38] that were adequate to describe the late-time universe.

\section{B. Reconstruction of cyclic evolution}

In this subsection we extend the above analysis in order to construct a sequence of bounces and turnarounds, namely in order to obtain a cyclic cosmological evolution. As a first step we will consider a specific cyclic scale factor and we will reconstruct the corresponding $\mathcal{K}$ and $\mathcal{G}$ forms that generate it. Then, having obtained information for their qualitative behavior, we will consider specific $\mathcal{K}$ and $\mathcal{G}$ forms and show that they lead to cyclic evolution. In the following paragraphs we examine two such cases separately.

\section{Model I: $\mathcal{K}=\phi / 2$ and $\mathcal{G}=\mathcal{G}(B)$}

As a first model we consider $\mathcal{K}=\phi / 2$ and $\mathcal{G}=\mathcal{G}(B)$. We start considering an oscillating scale factor of the form

$$
a(t)=A \sin (\omega t)+a_{c},
$$

with $a_{c}-A>0$ the scale factor at the bounce point, and $A+a_{c}$ the scale factor value at the turnaround. Inserting these into (11), (13) and (14), and replacing $\mathcal{G}_{B}(t)=\mathcal{G}^{\prime}(t) / B^{\prime}(t)$, and $\mathcal{G}_{B B}(t)=\left(\mathcal{G}^{\prime \prime}(t) B^{\prime}(t)-\right.$ $\left.B^{\prime \prime}(t) \mathcal{G}^{\prime}\right) /\left(B^{\prime}(t)\right)^{3}$, we obtain a system of differential equations for $\phi(t), \chi(t)$ and $G(t)$. We mention that since cyclic cosmology describes the whole universe evolution, and not only the phase around the bounce, we must necessarily include the matter sector. Without loss of generality we focus on the dust matter case, where $p_{m}=0$ and thus the continuity equation (16) leads to $\rho_{m}=\rho_{m b}\left(a_{c}-A\right)^{3} / a^{3}$.

Since the above system of differential equations cannot be solved analytically, we elaborate it numerically and we obtain the solutions for $\phi(t), \chi(t)$ and $G(t)$, and thus for $B(t)$ too, and therefore we acquire the function $\mathcal{G}(B)$ in a parametric form. In Fig. 5 we depict this reconstructed $\mathcal{G}(B)$, which is the one that gives rise to the cyclic scale factor (19).

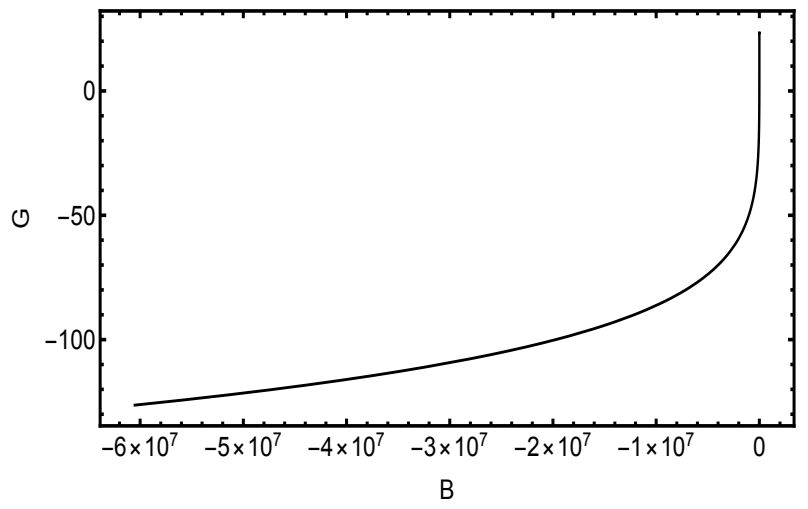

FIG. 5: The reconstructed $\mathcal{G}(B)$ that generates the cyclic scale factor 34, in the case where $\mathcal{K}=\phi / 2$. The parameters have been chosen as $A=0.05, a_{c}=10, \omega=1$. All quantities are measured in $M_{p l}$ units.

As we observe from the above procedure, and in particular from Fig. 5 , in order to acquire a cyclic scale factor in the case where $\mathcal{K}=\phi / 2$, we need a $\mathcal{G}(B)$ function that resembles an exponential function of $B$. Thus, we can now reverse the reconstruction procedure and impose an exponential form of $\mathcal{G}(B)$, namely

$$
\mathcal{G}(B)=G_{0} e^{G_{1} B},
$$

where $G_{0}$ and $G_{1}$ are parameters. Substituting it into (11), (12) and (13), with $\mathcal{K}=\phi / 2$, we obtain three second-order differential equations for $a(t), \phi(t)$ and $\chi(t)$. Elaborating the system numerically we extract the scale 


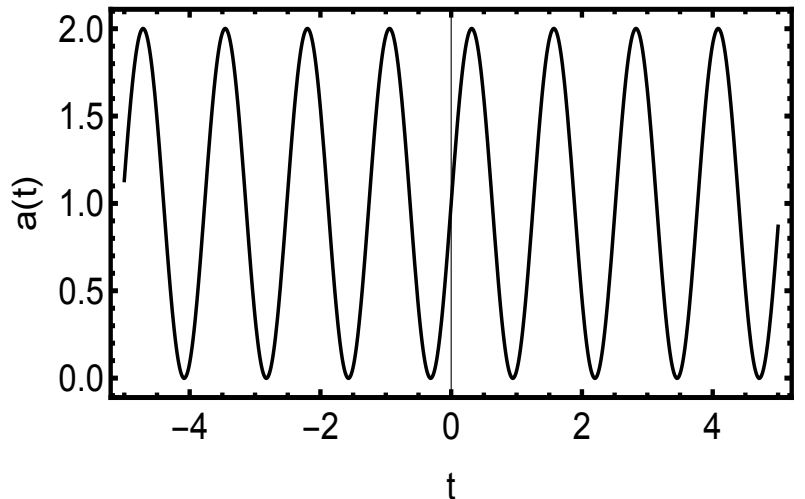

FIG. 6: The evolution of the scale factor a $(t)$ that is generated by the exponential form $\mathcal{G}(B)=G_{0} e^{G_{1} B}$, in the case where $\mathcal{K}=\phi / 2$, with $G_{0}=2.4$ and $G_{1}=0.8$ in $M_{p l}$ units.

factor, and we present it in Fig. 6. Thus, we can see that new gravitational scalar-tensor theories with $\mathcal{K}=\phi / 2$ and $\mathcal{G}(B)=G_{0} e^{G_{1} B}$ can indeed produce a cyclic universe. We mention here that the exponential $\mathcal{G}(B)$ may also lead to a single bounce realization, as we saw in the previous subsection, however what distinguishes the two possibilities are the parameter values.

\section{Model II: $\mathcal{K}=\phi / 2+f(B)$ and $\mathcal{G}=\xi B$}

We now study cyclicity in a different model, namely in the case where $\mathcal{K}=\phi / 2+f(B)$ and $\mathcal{G}=\xi B$, with $\xi$ a parameter. Similarly to the previous paragraph, we first consider the cyclic scale factor (34), in order to numerically reconstruct $f(B)$ and acquire a qualitative picture of its form. Substituting these into equations (11), (13) and (14), replacing $\mathcal{K}_{B}=f^{\prime}(t) / B^{\prime}(t)$ and $\mathcal{K}_{B B}(t)=\left(f^{\prime \prime}(t) B^{\prime}(t)-B^{\prime \prime}(t) f^{\prime}\right) /\left(B^{\prime}(t)\right)^{3}$, and considering a dust matter sector, we obtain a system of differential equations for $\phi(t), \chi(t)$ and $f(t)$. Solving it numerically we extract $\phi(t), \chi(t)$ and $f(t)$, and therefore $B(t)$ too, and thus we obtain the function $\mathcal{G}(B)$ in a parametric form. In Fig. 7 we show this reconstructed $\mathcal{G}(B)$, which is the one that gives rise to the cyclic scale factor (19).

As we observe from Fig. 7, in order to acquire a cyclic scale factor in the case where $\mathcal{K}=\phi / 2+f(B)$ and $\mathcal{G}=$ $\xi B$, we need a $f(B)$ function that resembles an parabolic function. Having this in mind we can now consider as an input the parabolic form

$$
\mathcal{K}(\phi, B)=\phi / 2+\left(B-B_{0}\right)^{2},
$$

with $B_{0}$ a constant. Inserting into (11), (12) and (13), with $\mathcal{G}=\xi B$, we obtain a system of differential equations for $a(t), \phi(t)$ and $\chi(t)$. Solving the equations numerically we extract the scale factor, and we depict it in Fig. 8 . Hence, we deduce that the theories at hand with $\mathcal{K}(\phi, B)=\phi / 2+\left(B-B_{0}\right)^{2}$ and $\mathcal{G}=\xi B$ can indeed produce a cyclic universe. Note that, as we saw in the

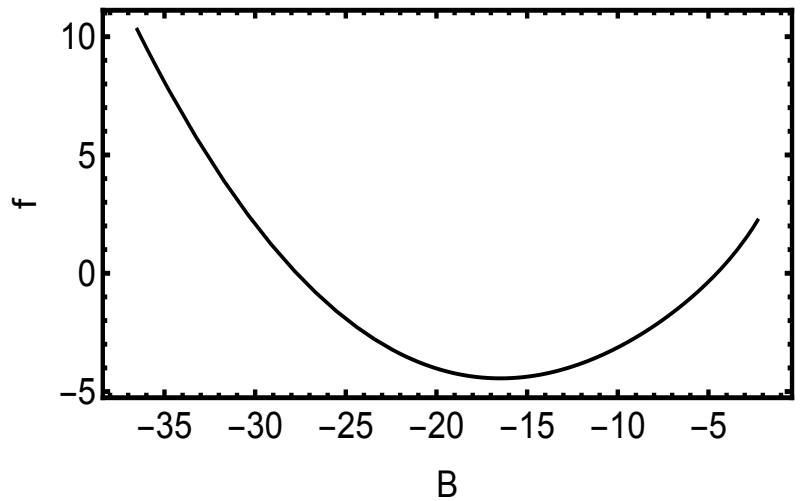

FIG. 7: The reconstructed $f(B)$ that generates the cyclic scale factor 34, in the case where $\mathcal{K}=\phi / 2+f(B)$ and $\mathcal{G}=\xi B$. The parameters have been chosen as $A=0.05, a_{c}=10, \omega=1$ and $\xi=0.1$. All quantities are measured in $M_{p l}$ units.

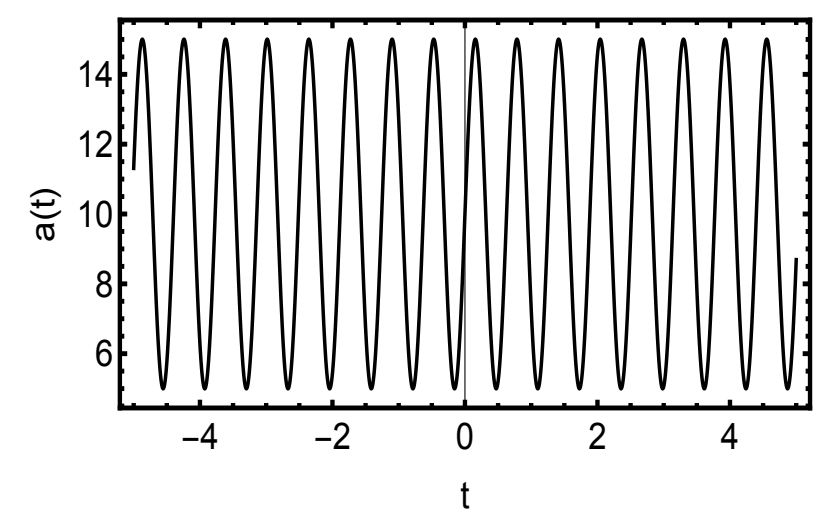

FIG. 8: The evolution of the scale factor a $(t)$ that is generated by $\mathcal{K}(\phi, B)=\phi / 2+\left(B-B_{0}\right)^{2}$ and $\mathcal{G}=\xi B$, with $B_{0}=19.7$ and $\xi=0.1$ in $M_{p l}$ units.

previous subsection, this parabolic form for $\mathcal{K}(\phi, B)$ may also lead to a single bounce solution, however what distinguishes the two possibilities are the parameter values.

\section{CONCLUSIONS}

In this work we investigated the bounce and cyclicity realization in the framework of new gravitational scalartensor theories. In particular, in these theories one considers a Lagrangian with the Ricci scalar and its first and second derivatives, however in a specific combination that makes the theory free of ghosts. Transforming into the Einstein frame, one can show that these constructions propagate $2+2$ degrees of freedom, and thus they are a subclass of bi-scalar extensions of general relativity. Nevertheless, the fact that these theories can be expressed in pure geometrical terms is a significant advantage.

We studied bouncing and cyclic solutions in various cases, reconstructing the forms of the functions $\mathcal{K}(\phi, B)$ and $\mathcal{G}(\phi, B)$ that can give rise to a given scale factor. Thus, having in mind the necessary qualitative form of 
these functions, we were able to reverse the procedure in the more physical base, namely we considered suitable simple functions $\mathcal{K}(\phi, B)$ and $\mathcal{G}(\phi, B)$ exhibiting this qualitative form, and we showed that they can give rise to a bounce or cyclic scale factor.

We close this work by referring to the perturbations of the obtained background solutions. In every bouncing scenario the analysis of perturbations is necessary, since they are related to observables such as the tensorto-scalar ratio. While in inflationary cosmology the generation of the primordial power spectrum requires that the cosmological fluctuations emerge initially inside the Hubble horizon, then they exit it, and later on they reenter, in a bounce scenario the situation is radically different. In particular, in bouncing cosmology the quantum fluctuations around the initial vacuum state are generated in the contraction phase before the bounce, they exit the Hubble radius as contraction continues, since the Hubble horizon decreases faster than the wavelengths of the primordial fluctuations, then the bounce happens, and finally they re-enter inside the horizon at later times in the expanding phase. Definitely, at the bounce point the background evolution could affect the perturbations scale dependence, however one expects this effect to be important only in the UV regime, where the gravitational modification effects play role, while the IR regime, which is responsible for the primordial perturbations related to the large-scale structure, remains almost unaffected 43 45. .

Although the generation of perturbations in bouncing models with one extra scalar degree of freedom is well understood and studied [34, 46, 47], in the case where the underlying theory has more than one extra scalar degrees of freedom, where both of them contribute to the bounce, the perturbation generation has not been stud- ied in detail. In particular, the examined scenarios in this subclass assume that one of the two extra scalar degrees of freedom is the dominant one at some point 48 53]. However, this approach cannot be straightforwardly followed in scenarios where both fields have more or less equal contribution to the bounce realization, and one can see that the present scenario lies in this category. Hence, the analysis of perturbation generation in the bouncing scenario at hand has to be performed in a thorough and systematic way, through the full and detailed perturbation generation analysis of general two-field bounces. For this investigation one could use concepts and techniques of the perturbation generation in two-field inflation [5458] (which is different from single-field inflation with a second sub-dominant field such as in cases of hybrid inflation 59 61]). Nevertheless, this detailed analysis of perturbation generation in two-field bouncing models is a separate work that lies beyond the scope of the present project, and it is left for a future investigation.

In summary, we showed that the new gravitational scalar-tensor theories, namely a subclass of bi-scalar extension of general relativity that can be constructed by pure geometrical terms, can naturally give rise to bouncing and cyclic behavior. This capability acts as an additional advantage for these theories.

\section{Acknowledgments}

This article is based upon work from COST Action "Cosmology and Astrophysics Network for Theoretical Advances and Training Actions", supported by COST (European Cooperation in Science and Technology).
[1] A. H. Guth, Phys. Rev. D 23, 347 (1981).

[2] A. Borde and A. Vilenkin, Phys. Rev. Lett. 72, 3305 (1994).

[3] V. F. Mukhanov and R. H. Brandenberger, Phys. Rev. Lett. 68, 1969 (1992).

[4] S. 'i. Nojiri and S. D. Odintsov, eConf C 0602061, 06 (2006) [Int. J. Geom. Meth. Mod. Phys. 4, 115 (2007)].

[5] S. Capozziello and M. De Laurentis, Phys. Rept. 509, 167 (2011).

[6] G. Veneziano, Phys. Lett. B 265, 287 (1991).

[7] J. Khoury, B. A. Ovrut, P. J. Steinhardt and N. Turok, Phys. Rev. D 64, 123522 (2001).

[8] J. Khoury, B. A. Ovrut, N. Seiberg, P. J. Steinhardt and N. Turok, Phys. Rev. D 65, 086007 (2002).

[9] K. Bamba, A. N. Makarenko, A. N. Myagky, S. Nojiri and S. D. Odintsov, JCAP 1401 (2014) 008.

[10] S. Nojiri and S. D. Odintsov, Mod. Phys. Lett. A 29, no. 40, 1450211 (2014).

[11] Y. -F. Cai, S. -H. Chen, J. B. Dent, S. Dutta and E. N. Saridakis, Class. Quant. Grav. 28, 215011 (2011).

[12] T. Biswas, A. Mazumdar and W. Siegel, JCAP 0603,
009 (2006).

[13] S. Nojiri and E. N. Saridakis, Astrophys. Space Sci. 347, 221 (2013).

[14] Y. Shtanov and V. Sahni, Phys. Lett. B 557, 1 (2003).

[15] E. N. Saridakis, Nucl. Phys. B 808, 224 (2009).

[16] Y. F. Cai and E. N. Saridakis, JCAP 0910, 020 (2009).

[17] E. N. Saridakis, Eur. Phys. J. C 67, 229 (2010).

[18] Y. -F. Cai and E. N. Saridakis, Class. Quant. Grav. 28, 035010 (2011).

[19] Y. F. Cai, C. Gao and E. N. Saridakis, JCAP 1210, 048 (2012).

[20] M. Bojowald, Phys. Rev. Lett. 86, 5227 (2001).

[21] S. D. Odintsov and V. K. Oikonomou, Phys. Rev. D 90, no. 12,124083 (2014).

[22] S. D. Odintsov, V. K. Oikonomou and E. N. Saridakis, Annals Phys. 363, 141 (2015).

[23] Y. F. Cai, T. Qiu, Y. S. Piao, M. Li and X. Zhang, JHEP 0710, 071 (2007).

[24] Y. -F. Cai, E. N. Saridakis, M. R. Setare and J. -Q. Xia, Phys. Rept. 493, 1 (2010).

[25] S. Nojiri, S. D. Odintsov, V. K. Oikonomou and 
E. N. Saridakis, JCAP 1509, 044 (2015).

[26] E. N. Saridakis and J. M. Weller, Phys. Rev. D 81, 123523 (2010).

[27] E. N. Saridakis and J. Ward, Phys. Rev. D 80, 083003 (2009).

[28] R. C. Tolman, Relativity, Thermodynamics and Cosmology, Oxford U. Press (1934).

[29] P. J. Steinhardt and N. Turok, Phys. Rev. D 65, 126003 (2002).

[30] P. J. Steinhardt and N. Turok, Science 296, 1436 (2002).

[31] J. E. Lidsey, D. J. Mulryne, N. J. Nunes and R. Tavakol, Phys. Rev. D 70, 063521 (2004).

[32] L. Baum and P. H. Frampton, Phys. Rev. Lett. 98, 071301 (2007).

[33] S. Nojiri, S. D. Odintsov and D. Saez-Gomez, AIP Conf. Proc. 1458, 207 (2011).

[34] M. Novello and S. E. P. Bergliaffa, Phys. Rept. 463, 127 (2008).

[35] F. Finelli and R. Brandenberger, Phys. Rev. D 65, 103522 (2002).

[36] Y. -F. Cai, W. Xue, R. Brandenberger and X. Zhang, JCAP 0905, 011 (2009).

[37] A. Naruko, D. Yoshida and S. Mukohyama, Class. Quant. Grav. 33, no. 9, 09LT01 (2016).

[38] E. N. Saridakis and M. Tsoukalas, Phys. Rev. D 93, no. 12,124032 (2016).

[39] G. W. Horndeski, Int. J. Theor. Phys. 10 (1974) 363.

[40] A. Nicolis, R. Rattazzi and E. Trincherini, Phys. Rev. D 79 (2009) 064036.

[41] C. Deffayet, G. Esposito-Farese and A. Vikman, Phys. Rev. D 79, 084003 (2009).

[42] J. Gleyzes, D. Langlois, F. Piazza and F. Vernizzi, Phys. Rev. Lett. 114, no. 21, 211101 (2015).

[43] L. Battarra, M. Koehn, J. L. Lehners and B. A. Ovrut,
JCAP 1407, 007 (2014).

[44] J. Quintin, Z. Sherkatghanad, Y. F. Cai and R. H. Brandenberger, Phys. Rev. D 92, no. 6, 063532 (2015).

[45] M. Koehn, J. L. Lehners and B. Ovrut, Phys. Rev. D 93, no. 10, 103501 (2016).

[46] L. E. Allen and D. Wands, Phys. Rev. D 70, 063515 (2004).

[47] T. Qiu, J. Evslin, Y. F. Cai, M. Li and X. Zhang, JCAP 1110, 036 (2011).

[48] V. Bozza and G. Veneziano, Phys. Lett. B 625, 177 (2005).

[49] F. Di Marco, F. Finelli and R. Brandenberger, Phys. Rev. D 67, 063512 (2003).

[50] V. Bozza and G. Veneziano, JCAP 0509, 007 (2005).

[51] H. S. Kim and J. c. Hwang, Phys. Rev. D 75, 043501 (2007).

[52] K. Koyama and D. Wands, JCAP 0704, 008 (2007).

[53] Y. F. Cai, E. McDonough, F. Duplessis and R. H. Brandenberger, JCAP 1310, 024 (2013).

[54] J. Garcia-Bellido and D. Wands, Phys. Rev. D 53, 5437 (1996).

[55] D. Wands, N. Bartolo, S. Matarrese and A. Riotto, Phys. Rev. D 66, 043520 (2002).

[56] F. Vernizzi and D. Wands, JCAP 0605, 019 (2006).

[57] Z. Lalak, D. Langlois, S. Pokorski and K. Turzynski, JCAP 0707, 014 (2007).

[58] C. M. Peterson and M. Tegmark, Phys. Rev. D 84, 023520 (2011).

[59] A. D. Linde, Phys. Rev. D 49, 748 (1994).

[60] J. Garcia-Bellido, A. D. Linde and D. Wands, Phys. Rev. D 54, 6040 (1996).

[61] D. H. Lyth, JCAP 0511, 006 (2005). 